MATHEMATICAL ASSOCIATION

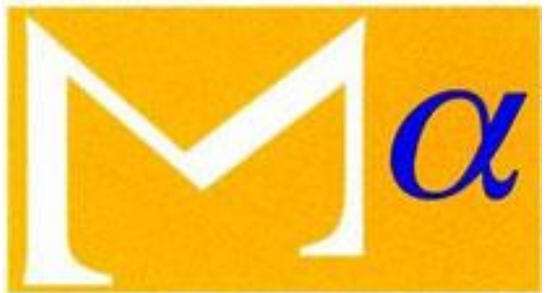

supporting mathematics in education

Extension of the Theory of Inversion to Conics

Author(s): J. J. Milne

Source: The Mathematical Gazette, Vol. 9, No. 141 (Jul., 1919), pp. 365-368

Published by: Mathematical Association

Stable URL: http://www.jstor.org/stable/3604481

Accessed: 30-11-2015 03:19 UTC

Your use of the JSTOR archive indicates your acceptance of the Terms \& Conditions of Use, available at http://www.jstor.org/page/ info/about/policies/terms.jsp

JSTOR is a not-for-profit service that helps scholars, researchers, and students discover, use, and build upon a wide range of content in a trusted digital archive. We use information technology and tools to increase productivity and facilitate new forms of scholarship. For more information about JSTOR, please contact support@jstor.org. 


\section{EXTENSION OF THE THEORY OF INVERSION TO CONICS.}

By The Rev. J. J. Milne, M.A.

[The references are to the author's treatise on Cross-Ratio Geometry.]

Let $t$ be the centre of a circle, radius $r, p$ any point in its plane, and draw the diameter through $p$. Then the inverse of $p$ is a point $p^{\prime}$ on $t p$ such that $t p . t p^{\prime}=r^{2}$. This circle is called the circle of inversion, its centre $t$ the pole of inversion and the square of its radius the constant of inversion, and each of the points $p, p^{\prime}$ is the inverse of the other. This relation is a metric one : a descriptive relation can be obtained as follows.

Every point on the polar of $p$ is conjugate to $p$, and the inverse of $p$ is that conjugate point which lies on the diameter through $p$; in other words, the inverse of a point is the intersection of its polar with the diameter through the point.

As long as we take a circle as our curve of inversion it is immaterial which of these relations we take as our base. Suppose, however, we take a conic as the fundamental curve of inversion, then it is evident that we cannot deal with the metrical relation, but there is nothing to prevent us from considering the question descriptively.

Now, two of the distinguishing features of a circle considered as a member of the family of conics are, (1) that it passes through the two circular points at infinity, usually denoted by $i, i^{\prime}$, and (2) that its centre is the pole of the line at infinity. In passing from the circle to the conic these imaginary points will be replaced by real points $I, I^{\prime}$, lying on the conic, and $t$, the centre of the circle, will become $T$, the pole of the chord $I I^{\prime}$.

As orthogonal circles enter largely into the theory of inversion, it may be as well to consider what they become in the case of conics. Two orthogonal circles possess the following fundamental properties, from any one of which the others may be derived:

(1) The tangents at a point of intersection are at right angles.

(2) The tangents at a point of intersection pass through the centres.

(3) The centre of each circle is the pole of the common chord for the other circle and the pole of $i i^{\prime}$ for its own circle.

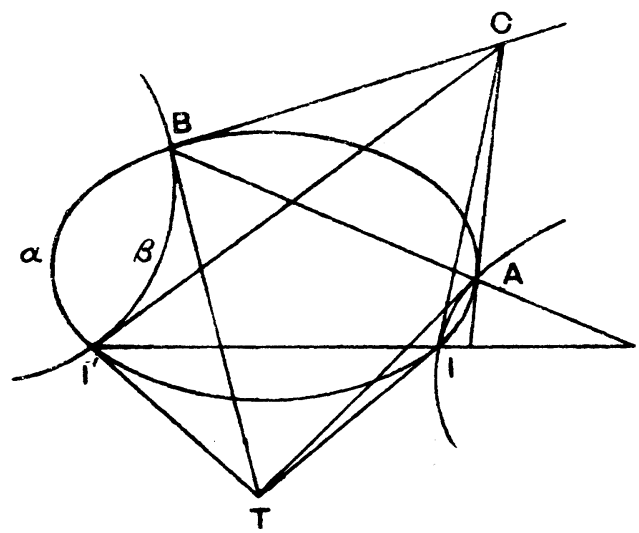

Frg. 1.

Now, let $\alpha, \beta$ be the two corresponding conics having $I I^{\prime}, A B$ for a pair of common chords, and let $T, C$ be the poles of $I I^{\prime}$ (Fig. 1). 
Then

(1) The tangents at $A$ divide $I I^{\prime}$ harmonically.

(2) The tangents at $A$ pass through $T, C$.

(3) $T$, the pole of $I I^{\prime}$ for $\alpha$, is the pole of $A B$ for $\beta$.

From (1), these conics may be called harmotomic; these and other important froperties relating to them are proved directly in Cross-Ratio Geometry, Cap. XIII.

When the curve of inversion is a circle there are three fundamental propositions, viz. (Fig. 2):

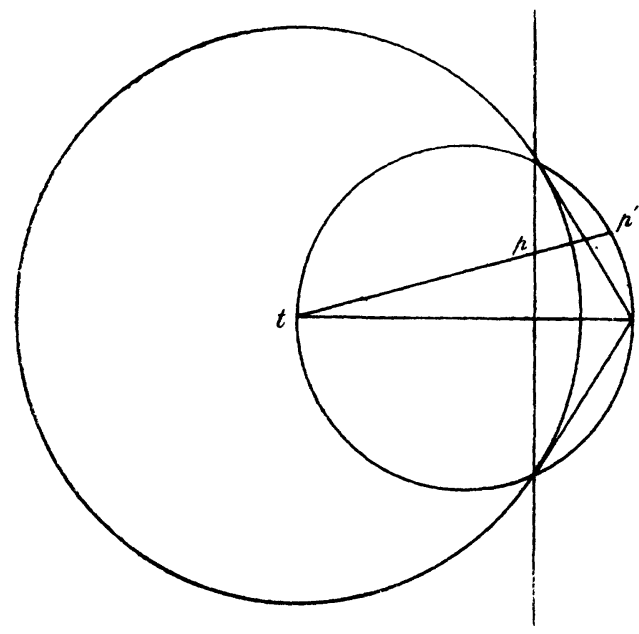

FI'i. 2.

(1) The inverse of a line is a circle which passes through the pole of inversion, the pole of the line, the points $i, i^{\prime}$, and the points where the line cuts the circle of inversion.

(2) The inverse of a circle which passes through the pole of inversion is a straight line.

(3) The inverse of a circle which does not pass through the pole of inversion is a circle coaxial with the other two.

Transform the circle of inversion into a conic $A$, and let $I, I^{\prime}$ represent the circular points. Then $T$, the pole of $I I^{\prime}$, will represent the centre $t$, and if $P, P^{\prime}$ represent the inverse points $p, p^{\prime}$, the point $P^{\prime}$ is the intersection of $T P$ with the polar of $P$. Then we may speak of the conic $A$ as the conic of inversion, the point $T$ as the pole of inversion, and $P^{\prime}$ as the inverse of $P$ for the point $T$, it being understood that the inversion is with respect to the conic $A$. Note. - It is obvious that $P$ is the inverse of $P^{\prime}$, and therefore $P$ and $P^{\prime}$ may be called inverse points.

We will now state and prove the three fundamental properties for the conic corresponding to the above for the circle (Fig. 3):

(1) The inverse of a straight line for a conic is a conic passing through six known points, viz. the pole of inversion, the pole of the given line, and the points where their polars intersect the conic of inversion. Let $T$ be the pole of inversion, $D E$ the given line, $F$ its pole. Let $H, K$ be two conjugate points on $D E$, and let $T H$ meet $F K$ in $P$. Thus $P, H$ are inverse points. Then, since the range $(H)=$ the range $(K)$,

$$
\therefore \text { the pencil } T(H)=\text { the pencil } F(K) \text {. }
$$


$\therefore$ the locus of $P$ is a conic through $T$ and $F$. The conic evidently also passes through $D, E, I I^{\prime}$. Another proof of this is given in $C .-R$. Art. 191 .

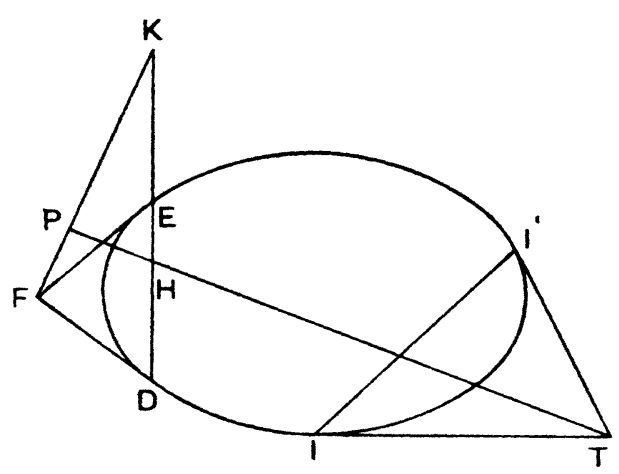

Fig. 3.

(2) The inverse of a conic passing through the pole of inversion $T$ and the points $I, I^{\prime}$ is a straight line, viz. the other common chord corresponding to $I I^{\prime}$. This is obvious from (1), since $P$ and $H$ are inverse points.

(3) If $A$ is the conic and $T$ the pole of inversion, the inverse of a conic $B$ which passes through $I$ and $I^{\prime}$ but not through $T$ is a conic $C$ of the pencil determined by $A$ and $B$. Let $P$ be any point on $B$ (Fig. 4), $I I^{\prime}$ one of the

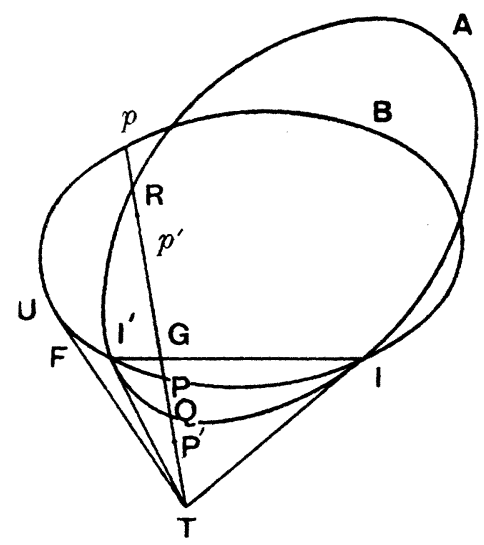

Frg. 4.

common chords of $A$ and $B, T$ the pole of $I I^{\prime}$ for $A$. Join $T P$, meeting $A$ in $Q, R, B$ again in $p$, and $I I^{\prime}$ in $G$. Let $P^{\prime}, p^{\prime}$ be the inverses of $P, p$.

Then

$$
\left(P P^{\prime} Q R\right)=-1=\left(p p^{\prime} Q R\right)=\left(p p^{\prime} R Q\right) \text {. }
$$

$\therefore$ by Art. 105, $\left(P p, P^{\prime} p^{\prime}, Q R\right)$ is an involution range.

$\therefore$ by Sturm's Theorem, Art. 216, $P^{\prime}, p^{\prime}$ are points on a conic $C$ of the pencil determined by $A$ and $B$.

Since

$$
(T G Q R)=-1=\left(P P^{\prime} Q R\right)=\left(p p^{\prime} Q R\right),
$$

$\therefore\left(P P^{\prime}, p p^{\prime}, T G\right)$ is an involution range.

$$
\therefore\left(T G P p^{\prime}\right)=\left(G T P^{\prime} p\right)=\left(T G p P^{\prime}\right)=\lambda \text {, }
$$


and if the five points $P, P^{\prime}, p, p^{\prime}, G$ are given, the sixth $T$ is given, and is unique. $\therefore T$ is the centre, $I I^{\prime}$ the axis and $\lambda$ the constant of homology of $B$ and $C$.

$\therefore$ by Art. 243, $B$ and $C$ are homologous, and by Art. 244, if $T U$ is the tangent to $B$, meeting $I I^{\prime}$ in $F$, the conic $C$ will touch $T U$.

If $T P_{1}^{\prime} Q_{1} P_{1} G_{1} p_{1} R_{1} p_{1}^{\prime}$ is any other transversal through $T$, we still have to show that $P_{1}^{\prime}, p_{1}^{\prime}$ are points on the same conic $C$ of the pencil as that on which $P^{\prime}, p^{\prime}$ lie. As above, we know that they will lie on a conic $C^{\prime}$ of the pencil, and that $B$ and $C^{\prime}$ are homologous conics having $T$ for centre and $I I^{\prime}$ for axis of homology, and $C^{\prime}$ will touch $T U$ by Art. 244 . But only one conic of the pencil besides $B$ can be drawn to touch $T U ; \therefore C^{\prime}$ coincides with $C$.

Cor. 1. The pole of inversion is a tangent vertex of a conic and its inverse conic.

Cor. 2. By the converse of Art. 248, if $M, N$ are the poles of $I I^{\prime}$ for $B$ and $C, M p$ and $N P^{\prime}$ intersect on $I I^{\prime}$, i.e. in the case of a circle and its inverse the radii corresponding to these lines are parallel.

If $P, P^{\prime}$ are inverse points, any conic through $P, P^{\prime}, I, I^{\prime}$ cuts the conic of inversion harmotomically. (Art. 205, Cor.)

Any conic $B$ can be inverted into itself by taking as the conic of inversion a conic $A$ which cuts it harmotomically, and if $A$ is the conic of inversion and a conic $B$ is inverted into itself, $A$ and $B$ are harmotomic.

Any two conics having the same common chord with the conic of inversion and cutting it harmotomically can be inverted into themselves. Let $\alpha, \alpha^{\prime}$ be any two conics, $I I^{\prime}, Q R$ a pair of common chords, on $Q R$ take any point $T$, join $T I$ and $T I^{\prime}$, draw $T A$ a tangent to $\alpha$. Then the conic $\beta$ passing through $A$ and touching $T I, T I^{\prime}$ at $I, I^{\prime}$ will be the conic of inversion and will be harmotomic to $\alpha$. And by Art. 205, Cor., $Q$ and $R$ being conjugate points for $\beta, \alpha^{\prime}$ is harmotomic to $\beta$. And by Art. 205, any transversal through $T$ cuts each of the conics $\alpha, \alpha^{\prime}$ in a pair of inverse points. Q.E.D.

If two conics are inverted into themselves, the line joining their poles for $I I^{\prime}$ inverts into a conic which cuts them both harmotomically.

A system of conics having double contact will invert into a pencil of conics, the pole of inversion and the pole of contact being two vertices of the common self-conjugate triangle of the pencil; and conversely, if a pencil of conics is inverted from a vertex of the self-conjugate triangle it inverts into a system of conics having double contact.

If we take as the pole of inversion one of the common points of the pencil, we obtain a pencil of straight lines; and conversely, the inverse of a pencil of lines is a pencil of conics passing through the pole of inversion and the centre of the pencil.

If any arbitrary point is taken as the pole of inversion, a pencil of conics will invert into another pencil of conics.

The theory of inversion was discovered for the circle in 1842 by Stubbs and Ingram. JoHN J. Milne.

27. E. Dr. Whewell has just published another book . . . on education, in which he cries up the geometrical processes in preference to analysis.- Sir John Herschel to Mrs. Somerville.-M. S.

28. F. Mr. Adams told Somerville that the following sentence in the sixth edition of the Connexion of the Physical Sciences, published in the year 1842, put it into his head to calculate the orbit of Neptune. "If after the lapse of years the tables formed from a combination of numerous observations should still be inadequate to represent the motions of Uranus, the discrepancies may reveal the existence, nay, even the mass and orbit of a body placed for ever beyond the sphere of vision."-M. S. 